\title{
TRANSPORTATION OF EURO PALLETS SOLVED AS A SPLIT DELIVERY VEHICLE ROUTING PROBLEM
}

\author{
Jan Fábry \\ ŠKODA AUTO University, Na Karmeli 1457, Mladá Boleslav, Czech Republic, EU, fabry@savs.cz
}

Keywords: split delivery vehicle routing problem, Euro pallet, vehicle routing problem, heuristics, CPLEX

Abstract: The article is aimed at the logistic problem of the company transporting Euro pallets to its customers. The main focus is on finding more effective routes for pallets distribution in terms of the total distance. The real instance can be solved as the vehicle routing problem. In many cases, splitting of the customer demand into several routes, can significantly improve the solution. The real problem consists in the pallets distribution from the depot to twelve customers. For solution, model in MPL for Windows is formulated and solved in CPLEX. Because of NP-hardness of the problem, it is necessary to propose heuristic algorithms for getting the effective feasible solution instead of unreachable optimum solution.

\section{Introduction}

In the Vehicle Routing Problem (VRP), capacitated vehicles must serve a set of customers with their demand quantities. In [1], mathematical models and methods are presented for many variations of VRP. Baldacci et al. [2] offer a survey of problems under vehicle and time window constraints. Because of NP-hardness of VRP, heuristic algorithms must be used for real instances [3]. In the Split Delivery Vehicle Routing Problem (SDVRP), customer demand can be splitted into several routes. The problem and corresponding savings were introduced in [4] and [5]. Applications and computational results can be found in [6]. Advanced heuristic algorithm is proposed in [7].

In the article, SDVRP with the identical capacitated vehicles, placed at one depot, is studied. The aim is to suggest the solution for the instance with twelve customers. The solution is compared with the existing strategy of the company and with the solution obtained from the optimization model of VRP.

Figure 1 shows the advantage of the formulation of the problem as SDVRP instead of VRP. In the example, the depot is located in node 1, customers are located in nodes 2,3 and 4 . In boxes, their demands are specified, the vehicle capacity is 10 units. Cost values are assigned to all arcs. While VRP offers 3 routes with the total cost of 60 , SDVRP offers 2 routes with total cost of 45 .

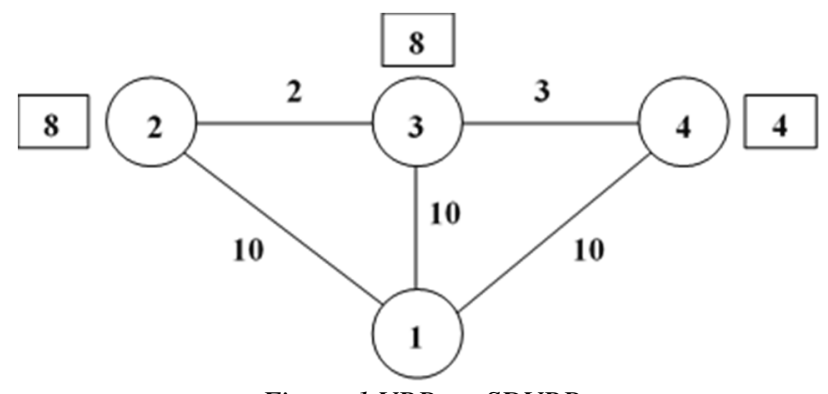

Figure 1 VRP vs. SDVRP

\section{Vehicle Routing Problem}

In the standard formulation of VRP, a number of vehicles located at one depot is supposed to be generally unlimited. They have the identical transportation capacity $V$. Let $n$ be a number of locations in the distribution network, depot is placed at location 1. Demand of customer $i$ is denoted by $q_{i}<V(i=2,3, \ldots, n)$. For each pair of locations, their distance is given as $c_{i j}(i, j=1,2, \ldots, n)$. Binary variable $x_{i j}$ is equal to 1 , if a vehicle visits location $j$ after visiting location $i, 0$ otherwise. Mathematical model of VRP originates from [1]:

$$
\begin{aligned}
& z=\sum_{i=1}^{n} \sum_{j=1}^{n} c_{i j} x_{i j} \rightarrow \min , \\
& \sum_{j=1}^{n} x_{i j}=1, \quad i=2,3, \ldots, n, \\
& \sum_{i=1}^{n} x_{i j}=1, \quad j=2,3, \ldots, n,
\end{aligned}
$$

$$
\begin{gathered}
u_{i}+q_{j}-V\left(1-x_{i j}\right) \leq u_{j}, \\
i=1,2, \ldots, n, \quad j=2,3, \ldots, n, \quad i \neq j, \\
u_{i} \leq V, \quad i=2,3, \ldots, n, \\
u_{1}=0, \\
x_{i j} \in\{0,1\}, \quad i, j=1,2, \ldots, n, \\
u_{i} \in R_{0}^{+}, \quad i=1,2, \ldots, n .
\end{gathered}
$$

The objective function (1) aims at minimizing total distance. Respecting constraints (2) and (3), each customer 
is visited exactly ones. Inequalities (4), containing variables $u_{i}$, are load balance constraints. Each route must respect vehicle capacity (5). At the depot, vehicle load is set to 0 according to (6). Although model (1) - (8) is proposed to pick up problem, it can be used for delivery problems. However, we must be careful about the interpretation of obtained results, especially values of load variables.

\section{Split Delivery Vehicle Routing Problem}

If there is at least one demand exceeding the vehicle capacity, it is necessary to solve the problem as the SDVRP. Because of the computational complexity of the problem, in real instances it is often necessary to use heuristic algorithms. First, the exact approach is offered.

\subsection{Mathematical model}

In the model of SDVRP, third index $k$ must be introduced for the identification of the vehicle which serves the customer. Variable $x_{i j}^{k}$ is equal to 1 , if the vehicle $k$ goes from location $i$ to location $j, 0$ otherwise. Variable $Q_{i}^{k}$ is associated with the part of demand of customer $i$ delivered by vehicle $k$. Let us suppose $K$ vehicles located at the depot. Then, the mathematical model of SDVRP, based on [7], can be formulated as follows:

$$
\begin{gathered}
z=\sum_{k=1}^{K} \sum_{i=1}^{n} \sum_{j=1}^{n} c_{i j} x_{i j}^{k} \rightarrow \min , \\
\sum_{j=2}^{n} x_{1 j}^{k} \leq 1, \quad k=1,2, \ldots, K, \\
\sum_{i=1}^{n} x_{i j}^{k}=\sum_{i=1}^{n} x_{j i}^{k}, \\
j=2,3, \ldots, n, \quad k=1,2, \ldots, K, \\
u_{i}^{k}+Q_{j}^{k}-V\left(1-x_{i j}^{k}\right) \leq u_{j}^{k}, \quad i=1,2, \ldots, n, \\
j=2,3, \ldots, n, \quad i \neq j, \quad k=1,2, \ldots, K, \\
u_{1}^{k}=0, \quad k=1,2, \ldots, K, \\
0 \leq Q_{i}^{k} \leq u_{i}^{k} \leq V, \\
i=2,3, \ldots, n, \quad k=1,2, \ldots, K, \\
\sum_{k=1}^{K} Q_{i}^{k}=q_{i}, \quad i=2,3, \ldots, n,
\end{gathered}
$$

$$
\begin{aligned}
& 0 \leq Q_{i}^{k} \leq q_{i} \sum_{j=1}^{n} x_{i j}^{k}, \\
& i=2,3, \ldots, n, \quad k=1,2, \ldots, K, \\
& x_{i i}^{k}=0, \\
& i=1,2, \ldots, n, \quad k=1,2, \ldots, K, \\
& x_{i j}^{k} \in\{0,1\}, \\
& i, j=1,2, \ldots, n, \quad k=1,2, \ldots, K .
\end{aligned}
$$

Similarly, to model of VRP, the objective function (9) represents the total length of all routes. Inequalities (10) allow each vehicle to leave the depot maximally once. Equations (11) assure that the vehicle entering any location must also leave it. Compared to the model of standard VRP, balancing constraints (12) contain load variables with additional index $k$. Using (13), all vehicle loads at the depot are set to 0 . Because all loads on each route must respect vehicle capacity, inequalities (14) are introduced. Equations (15) assure that partial deliveries will meet demand of each customer. Constraints (16) have two meanings. If vehicle $k$ does not serve customer $i$, i.e. there is no travel of this vehicle from the location of this customer, the corresponding partial delivery $Q_{i}^{k}$ must equal to 0 . On the contrary, if partial delivery is positive for vehicle $k$, it must leave customer $i$ on this route.

\subsection{Heuristic algorithm}

In [5] the following approach is proposed. At the beginning, it is necessary to find any feasible solution of VRP, i.e. the solution without splits. Such solution can be found using any heuristic algorithm described in [1] or using the mathematical model (1) - (8). Let us suppose, there are three routes in the solution. The vehicle on the first route has free capacity $V_{1}$, and the vehicle on the second route has free capacity $V_{2}$. On the third route, there is customer $s$, for which demand $q_{s}$ it is given:

$$
q_{s} \leq V_{1}+V_{2}
$$

i.e. demand can be splitted into two routes mentioned above.

Further, let us denote, in the original solution, $i_{1}$ and $j_{1}$ two successive locations on the first route, $i_{2}$ and $j_{2}$ two successive locations on the second route, $i_{3}$ immediate predecessor of location $s$ and $j_{3}$ its immediate successor (Fig. 2).

The sum of all distances related to the above-defined locations can be expressed as follows: 


$$
z_{1}=c_{i_{1} j_{1}}+c_{i_{2} j_{2}}+c_{i_{3} s}+c_{s j_{3}} .
$$

On Figure 2, the corresponding travels are highlighted in bold arrows.

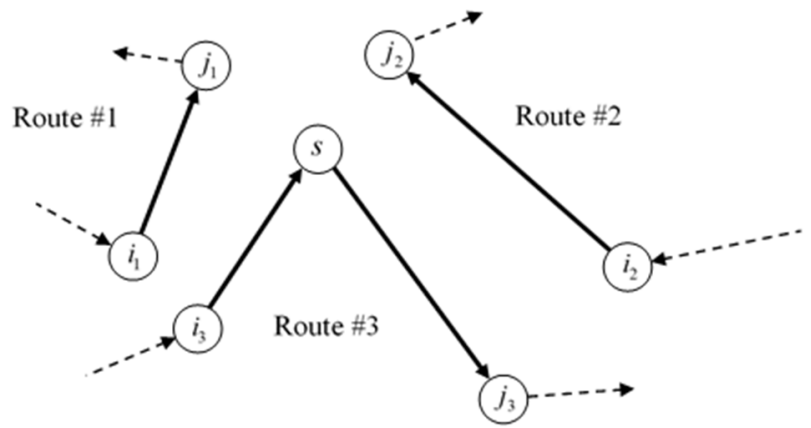

Figure 2 Solution without splits

Respecting (19), let us split demand $q_{s}$ into routes \#1 and \#2 (Fig. 3). New sum of distances is calculated as:

$$
z_{2}=c_{i_{1} s}+c_{s j_{1}}+c_{i_{2} s}+c_{s j_{2}}+c_{i_{3} j_{3}} .
$$

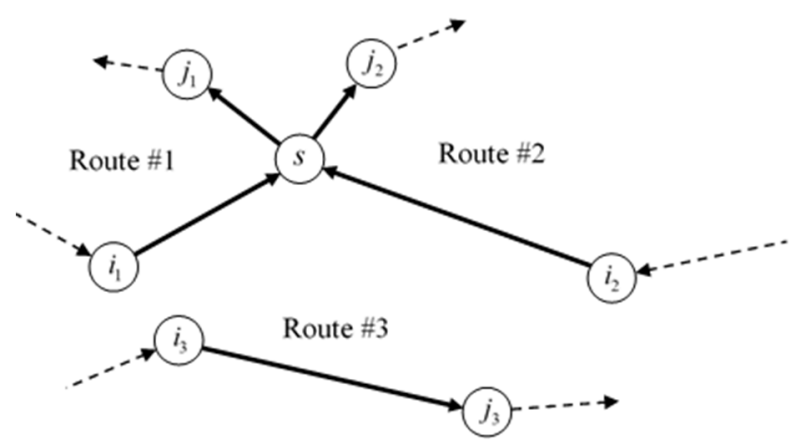

Figure 3 Solution with splits
If $\Delta z=\left(z_{1}-z_{2}\right)>0$, proposed change brings savings in distance and it is realized. This procedure can be repeated while the solution can be improved in terms of the total length of all routes.

\section{Application}

The main goal of the analysis is to solve the logistic problem of the company transporting Euro pallets to its customers. In the real instance of the company located in Bystrany, twelve customers are located in north-western region of Bohemia and Saxony. Demands of customers are listed in Table 1. For the transportation, the vehicle with the capacity of 70 pallets is used.

Table 1 Demand of customers

\begin{tabular}{lc} 
Location & Demand \\
\hline Bystrany & - \\
Teplice & 45 \\
Decin & 33 \\
Roudnice nad Labem & 85 \\
Kostany & 17 \\
Usti nad Labem & 19 \\
Bilina & 26 \\
Litvinov & 37 \\
Zim & 11 \\
Most & 28 \\
Drazdany & 89 \\
Dubi & 12 \\
Louny & 16
\end{tabular}

In Table 2, distances (in $\mathrm{km}$ ) between all pairs of locations can be found.

\begin{tabular}{cccccccccccccc} 
Location & 1 & 2 & 3 & 4 & 5 & 6 & 7 & 8 & 9 & 10 & 11 & 12 & 13 \\
\hline 1 & 0 & 4.9 & 42.8 & 43.4 & 11.6 & 22.2 & 17.5 & 28.5 & 12.3 & 33.4 & 70.0 & 10.8 & 38.7 \\
2 & 4.9 & 0 & 44.4 & 46.3 & 7.7 & 18.5 & 12.6 & 23.5 & 15.2 & 29.1 & 71.0 & 5.9 & 34.7 \\
3 & 42.8 & 44.4 & 0 & 65.0 & 53.0 & 25.9 & 59.3 & 68.8 & 39.8 & 66.6 & 71.5 & 50.7 & 72.8 \\
4 & 43.4 & 46.3 & 65.0 & 0 & 51.1 & 48.1 & 53.6 & 66.0 & 31.1 & 57.6 & 97.1 & 47.9 & 55.4 \\
5 & 11.6 & 7.7 & 53.0 & 51.1 & 0 & 27.2 & 15.3 & 17.5 & 23.4 & 29.9 & 70.9 & 5.3 & 37.4 \\
6 & 22.2 & 18.5 & 25.9 & 48.1 & 27.2 & 0 & 31.4 & 46.6 & 13.9 & 44.1 & 66.2 & 28.5 & 53.9 \\
7 & 17.5 & 12.6 & 59.3 & 53.6 & 15.3 & 31.4 & 0 & 28.3 & 22.5 & 15.9 & 83.0 & 19.4 & 22.1 \\
8 & 28.5 & 23.5 & 68.8 & 66.0 & 17.5 & 46.6 & 28.3 & 0 & 36.2 & 12.4 & 84.9 & 18.1 & 35.5 \\
9 & 12.3 & 15.2 & 39.8 & 31.1 & 23.4 & 13.9 & 22.5 & 36.2 & 0 & 43.2 & 66.0 & 18.1 & 43.6 \\
10 & 33.4 & 29.1 & 66.6 & 57.6 & 29.9 & 44.1 & 15.9 & 12.4 & 43.2 & 0 & 96.4 & 29.6 & 33.7 \\
11 & 70.0 & 71.0 & 71.5 & 97.1 & 70.9 & 66.2 & 83.0 & 84.9 & 66.0 & 96.4 & 0 & 66.8 & 106.5 \\
12 & 10.8 & 5.9 & 50.7 & 47.9 & 5.3 & 28.5 & 19.4 & 18.1 & 18.1 & 29.6 & 66.8 & 0 & 39.7 \\
13 & 38.7 & 34.7 & 72.8 & 55.4 & 37.4 & 53.9 & 22.1 & 35.5 & 43.6 & 33.7 & 106.5 & 39.7 & 0
\end{tabular}

In existing solution, six routes are realized with the total length of $683.7 \mathrm{~km}$ (Tab. 3). Deliveries are splitted in Teplice, Bilina, Roudnice nad Labem, Usti nad Labem and
Drazdany. While in case of Roudnice nad Labem and Drazdany it is necessary to split delivery, for other locations the splitting is optional. 


\begin{tabular}{|c|c|c|c|c|c|c|}
\hline \multicolumn{7}{|c|}{ Table 3 Existing solution } \\
\hline Route & 1 & 2 & 3 & 4 & 5 & 6 \\
\hline \multirow{6}{*}{ Locations } & Bystrany & Bystrany & Bystrany & Bystrany & Bystrany & Bystrany \\
\hline & Teplice & Bilina & Kostany & Usti & Drazdany & Drazdany \\
\hline & Bilina & Most & Dubi & Roudnice & Bystrany & Decin \\
\hline & Louny & Litvinov & Teplice & Zim & & Usti \\
\hline & Roudnice & Bystrany & Bystrany & Bystrany & & Bystrany \\
\hline & Bystrany & & & & & \\
\hline Total length & 138.4 & 74.3 & 27.7 & 113.7 & 140.0 & 189.6 \\
\hline Total load & 69 & 69 & 70 & 70 & 70 & 70 \\
\hline
\end{tabular}

Although the occupancy of vehicles is perfect, the company's management examined whether designed routes are cost-effective. For the application of mathematical models, the system MPL for Windows 5.0 and solver CPLEX 11 were used. Heuristics were designed in VBA for Excel. All calculations were executed in the Intel Core i7 Processor, $2.3 \mathrm{GHz}$, with $8 \mathrm{~GB}$ of RAM, running Windows 10, 64-bit OS. All results, compared with the existing solution, are listed in Table 4. Firstly, the optimum solution of VRP (1) - (8) was found with 2 direct fully-loaded-travels to Roudnice nad Labem and Drazdany. Of course, this in-advanced splitting (specifically $70+15$ and $70+19$ ) could be changed and investigated. The total length of all routes is $693.9 \mathrm{~km}$, i.e. approximately $10 \mathrm{~km}$ worse than the existing solution.

Then, the model of SDVRP (9) - (18) was applied. Due to the computational complexity of the problem, the optimal solution was not reached even after 24 hours of run. The objective value of the best found feasible solution is $680.5 \mathrm{~km}$, with the lower bound of $635 \mathrm{~km}$. Finally, above-suggested heuristic algorithm was applied for the feasible solution obtained using Clarke and Wright savings heuristic [8]. Both heuristics were developed in VBA for Excel. The total length of all routes is $712.2 \mathrm{~km}$.

\section{Result, discussion and conclusion}

For solution of real instance with the transportation of Euro pallets, various approaches were applied. If direct travels are used for customers with the demand exceeding the vehicle capacity, remaining deliveries can be included in routes optimized as VRP without possible splitting. More effective access is the application of mathematical model of SDVRP. However, the computational complexity of the problem is the obstacle to obtaining optimal solution even for small-sized real instances. Therefore, heuristic algorithms are used for such situations.

For comparison of all solutions to analysed instance, see Table 4. Although the optimality of the solution obtained by the application of model SDVRP cannot be proved, the solution might be optimal. Anyway, it is the best found solution (Tab. 5) which length is more than 3 $\mathrm{km}$ shorter than the existing solution. With the comparison of the solution obtained using model VRP, there are also two fully-loaded-travels to Drazdany and Roudnice nad Labem. However, the total length of all routes is more than $13 \mathrm{~km}$ shorter what shows the advantage of splitting, even in cases the vehicle capacity is higher than demand of each customer (optional splitting in Teplice and Usti nad Labem)

\begin{tabular}{ccccc} 
& $\begin{array}{c}\text { Table 4 Comparison of solutions } \\
\text { Company's } \\
\text { solution }\end{array}$ & $\begin{array}{c}\text { Model } \\
\text { VRP }\end{array}$ & $\begin{array}{c}\text { Model } \\
\text { SDVRP }\end{array}$ & $\begin{array}{c}\text { Heuristic } \\
\text { SDVRP }\end{array}$ \\
\hline Total length & 683.7 & 693.9 & 680.5 & 712.2 \\
Number of routes & 6 & 7 & 6 & 6
\end{tabular}

\begin{tabular}{|c|c|c|c|c|c|c|}
\hline \multicolumn{7}{|c|}{ Table 5 Best found solution } \\
\hline Route & 1 & 2 & 3 & 4 & 5 & 6 \\
\hline \multirow{8}{*}{ Locations } & Bystrany & Bystrany & Bystrany & Bystrany & Bystrany & Bystrany \\
\hline & Drazdany & Roudnice & Teplice & Teplice & Teplice & Usti \\
\hline & Bystrany & Bystrany & Dubi & Litvinov & Bilina & Decin \\
\hline & & & Kostany & Most & Louny & Drazdany \\
\hline & & & Bystrany & Bystrany & Roudnice & Bystrany \\
\hline & & & & & Zim & \\
\hline & & & & & Usti & \\
\hline & & & & & Bystrany & \\
\hline Total length & 140.0 & 86.8 & 27.7 & 74.2 & 162.2 & 189.6 \\
\hline Total load & 70 & 70 & 68 & 70 & 70 & 70 \\
\hline
\end{tabular}

The solution of real problem can be improved introducing vehicles with different capacity [9]. In such situation, cost evaluation of vehicles must be defined in the objective function. 


\section{References}

[1] TOTH, P., VIGO, D.: The Vehicle Routing Problem, SIAM Monographs on Discrete Mathematics, Vol. 9, SIAM Philadelphia, 2002.

[2] BALDACCI, R., TOTH, P., VIGO, D.: Exact algorithms for routing problems under vehicle capacity constraints, Annals of Operations Research, Vol. 175, No. 1, pp. 213-245, 2010.

[3] PISINGER, D., ROPKE, S.: A general heuristic for vehicle routing problems, Computers and Operations Research, Vol. 34, No. 8, pp. 2403-243, 2007.

[4] DROR, M., TRUDEAU, P.: Split delivery routing, Naval Research Logistics, Vol. 37, No. 3, pp. 383-402, 1990.

[5] DROR, M., TRUDEAU, P.: Savings by split delivery routing, Transportation Science, Vol. 23, No. 2, pp. 141-145, 1989.

[6] CHEN, S., GOLDEN, B., WASIL, E.: The split delivery vehicle routing problem: Applications, algorithms, test problems, and computational results, Networks, Vol. 49, No. 4, pp. 318-329, 2007.

[7] ARCHETTI, C., SPERANZA, M. G., HERTZ, A.: A tabu search algorithm for the split delivery vehicle routing problem, Transportation Science, Vol. 40, No. 1, pp. 64-73, 2006.

[8] LAPORTE, G., SEMET, F.: Classical Heuristics for the Capacitated VRP, In: TOTH, P., VIGO, D.: The Vehicle Routing Problem, SIAM Monographs on Discrete Mathematics, Vol. 9, SIAM Philadelphia, 2002.

[9] IMRAN, A., SALHI, S., WASSAN, N. A.: A variable neighbourhood-based heuristic for the heterogeneous fleet vehicle routing problem, European Journal of Operational Research, Vol. 197, pp. 509-518, 2009.

\section{Review process}

Single-blind peer review process. 